\title{
When the Tab Comes Due: Challenges in the Cost Structure of Browser Tab Usage
}

\author{
Joseph Chee Chang \\ josephcc@cs.cmu.edu \\ Carnegie Mellon University \\ Pittsburgh, Pennsylvania, USA \\ Julina Coupland \\ julina@gmail.com \\ Carnegie Mellon University \\ Pittsburgh, Pennsylvania, USA
}

\author{
Nathan Hahn \\ nhahn@cs.cmu.edu \\ Carnegie Mellon University \\ Pittsburgh, Pennsylvania, USA \\ Bradley Breneisen \\ bbreneis@andrew.cmu.edu \\ Carnegie Mellon University \\ Pittsburgh, Pennsylvania, USA
}

\author{
Yongsung Kim \\ yongsung@cmu.edu \\ Carnegie Mellon University \\ Pittsburgh, Pennsylvania, USA \\ Hannah S Kim \\ hkim122@gmail.com \\ Carnegie Mellon University \\ Pittsburgh, Pennsylvania, USA
}

\author{
John Hwong \\ Hwong.john@gmail.con \\ Carnegie Mellon University \\ Pittsburgh, Pennsylvania, USA
}

\author{
Aniket Kittur \\ nkittur@cs.cmu.edu \\ Carnegie Mellon University \\ Pittsburgh, Pennsylvania, USA
}

\begin{abstract}
Tabs have become integral to browsing the Web yet have changed little since their introduction nearly 20 years ago. In contrast, the internet has gone through dramatic changes, with users increasingly moving from navigating to websites to exploring information across many sources to support online sensemaking. This paper investigates how tabs today are overloaded with a diverse set of functionalities and issues users face when managing them. We interviewed ten information workers asking about their tab management strategies and walk through each open tab on their work computers four times over two weeks. We uncovered competing pressures pushing for keeping tabs open (ranging from interaction to emotional costs) versus pushing for closing them (such as limited attention and resources). We then surveyed 103 participants to estimate the frequencies of these pressures at scale. Finally, we developed design implications for future browser interfaces that can better support managing these pressures.
\end{abstract}

\section{CCS CONCEPTS}

- Human-centered computing $\rightarrow$ User models; Empirical studies in $\mathrm{HCI}$; ・ Information systems $\rightarrow$ Browsers.

\section{KEYWORDS}

world wide web, user behavior, browser interfaces, tabbed browsing, interviews, survey

\section{ACM Reference Format:}

Joseph Chee Chang, Nathan Hahn, Yongsung Kim, Julina Coupland, Bradley Breneisen, Hannah S Kim, John Hwong, and Aniket Kittur. 2021. When the

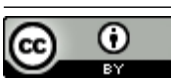

This work is licensed under a Creative Commons Attribution International 4.0 License.

CHI '21, May 8-13, 2021, Yokohama, Japan

(c) 2021 Copyright held by the owner/author(s).

ACM ISBN 978-1-4503-8096-6/21/05.

https://doi.org/10.1145/3411764.3445585
Tab Comes Due: Challenges in the Cost Structure of Browser Tab Usage. In CHI Conference on Human Factors in Computing Systems (CHI '21), May 8-13, 2021, Yokohama, Japan. ACM, New York, NY, USA, 15 pages. https: //doi.org/10.1145/3411764.3445585

\section{INTRODUCTION}

Tabs have become an integral part of how people browse and navigate the Web since they were introduced in the early 2000s, and they are now a ubiquitous feature in all major web browsers. As the Web becomes all-encompassing with complex applications and rich information, browser tabs have also become the portals for how people access information and make sense of the world today. People open browser tabs to check email inboxes, control music and video players, stash articles to read later, chat with friends, organize reviews and articles to plan trips, compare products, and research to write articles. However, while the Web has gone through dramatic changes in size, complexity, and usage, tabbed browsing interfaces remain largely the same. Tabs continue to be instantiated as simple temporally-ordered lists of independent pages with limited contextual cues and opportunities for manipulation. Indeed, the fixation on tabs as a metaphor is so strong that the most popular changes to using tabs involve relatively small design adjustments, such as making them a vertical instead of horizontal list [18, 25], building tabs of tabs [65], or saving sets of tabs as archived sessions [19]. In summary, there appears to be a disconnect between the increasing scope and complexity of users' online activities, and the design of tabbed browsing that we aim to explore in this paper.

As a result of this disconnect, recent anecdotal and empirical evidence suggests that tabbed browser interfaces now engender significant challenges, popularly referred to as "tab overload" [28$30,50,57,58]$. This includes difficulty in re-finding specific tabs, uncertainty in whether to keep open or to close tabs, being constantly distracted, harming productivity, causing feelings of stress and clutter, and, in extreme cases, causing users' browsers or computers to slow or crash. Most recently, empirical evidence from a survey conducted in 2019 showed that around half $(50.7 \%, \mathrm{~N}=75)$ of 
their participants considered tab clutter a problem [44]. Building on this, we conducted a preliminary survey to collect more empirical evidence about tab clutter ( $\mathrm{N}=64$, Age: $19-67 ; \mathrm{M}=33.7 ; \mathrm{SD}=10.6$; $57 \%$ male; $77 \%$ from the US). Based on self-reporting, $59 \%$ of our participants agreed that if they went through their browser tabs at the time when the survey took place, they would find some that should be closed. At the same time, only $19 \%$ agreed that laziness contributed to not closing them in the first place, and more than half $(55 \%)$ of the participants agreed that they feel like they can't let go of their tabs. These results suggest that there are alternative factors to a simplistic laziness explanation causing users to keep tabs open even though they were no longer useful and potentially causing adverse effects. For example, we found that having too many tabs opened can harm productivity with $28 \%$ of participants $(\mathrm{N}=64)$ agreeing that they often struggle with finding tabs they needed in their browser, and $25 \%$ of the participants reported that they had experienced browsers or computers crashing from having too many browser tabs. Finally, 30\% of our participants agreed that they "have a tab hoarding problem," echoing a common sentiment found more generally in digital file preservation [71]. Given that tabs are now ubiquitous to nearly all Web browsing, these numbers potentially translate to very large segments of the general population.

Despite the mounting evidence that the original browser tab paradigm is not well supporting a significant segment of users today in their online activities, there has been surprisingly little work on either understanding the causes or developing novel paradigms for evolving or replacing tabs. The most recent in-depth study on tabbed browsing behavior was conducted a decade ago by Dubroy et al. with Firefox users [15]. Yet at the time browsers that supported tabbed browsing only accounted for around $50 \%$ of the market share [72]. Further, their primary focus was to understand the growing popularity of tabbed browsing and its benefits compared to only using multiple browser windows [15]. Other papers exploring tab usage have similarly examined why tabs are used in place of previous interaction paradigms, such as the back button [15, 37, 38]. We believe a better understanding of the limitations of tabbed browsing today could spur novel interaction paradigms that could better support the modern Web. In this paper, we set out to provide an updated understanding on tabbed browsing behavior by characterizing the set of functions that tabs currently serve, often sub-optimally. We focus our investigation on the pressures that tabs put on users with the goal of developing a generative framework of tab functional requirements that can capture why current approaches are insufficient, and to guide the evolution of future tab-related interaction paradigms.

To accomplish this, we first conducted in-person interviews with ten information workers at two research facilities. We explored the issues they encountered when engaged in research and information work to build up a deep qualitative understanding of the challenges of tabbed browsing today. We then conducted an online survey to further investigate the frequencies of phenomena we observed in the in-person interviews. At a high level, we characterized various positive and negative drivers that governed participants' behavior. Overall, these drivers could be classified as two opposing forces: pressures to close tabs and pressures to keep tabs open. We found strong evidence that participants had numerous reasons to close their tabs, ranging from limited attention to limited browser resources to self-presentation. At the same time, we found a diverse set of reasons why it was not easy to close open tabs. These confirmed previously reported drivers observed a decade ago, such as reminding users of unfinished tasks [15], but also new factors relating to the cost structure of tabbed browsing, such as the cost of re-accessing pages, the sunk costs of finding and organizing information, the benefits of supporting an (unrealistic) aspirational self, and the uncertainty of the expected value of information in the future. These pressures to close vs. keep open tabs interact to create feelings of stress, being overwhelmed, and even shamefulness in our participants. Finally, based on our user model we conducted exploratory interviews with additional participants, probing potential solutions to develop a generative framework for future browser interfaces that can better support the underlying drivers behind current tabbed browsing behaviors.

\section{RELATED WORK}

\subsection{Managing and Revisiting Documents}

Assisting users in organizing and accessing multiple digital documents has long been studied across different domains since before tabbed browsing of Web documents gained wide popularity. Specifically, early research in the 1990s explored interfaces that can help users to save webpages as a collection of bookmarks and organize them. This was motivated by research that showed people frequently (up to $60 \%$ of all page loads according to [64]) revisited the same URLs, and characterized the Web in the 90s as a "recurrent system" [64]. One major thread of research explored interfaces that allowed users to organize bookmarks in 3D workspaces, such as WebBook [5] and DataMountain [59]. They found that by exploiting users' spatial memory, users were able to navigate to specific documents efficiently. Similar concepts were also explored in the context of programming environments in the 2010s with "canvas-based editors" [34] that allowed developers to arrange multiple program files and code snippets on 2D canvases for cross-referencing [4, 13, 34]. At a high level, the primary goal of these systems was to allow users to efficiently re-find specific documents within a familiar collection (i.e., collected bookmarks or existing codebases).

Conversely, a user's collection of browser tabs can be more ephemeral. Tabs serve a wide array of functionalities beyond revisitation and may contain information and documents unfamiliar to the user. Prior work has suggested that allowing users to freely arrange their documents in such scenarios can actually be detrimental to users [34]. Specifically, the flexibility of freely arranging documents in a $2 \mathrm{D}$ or $3 \mathrm{D}$ space could become obstacles when users were not already familiar with the information space to arrange them [42] or when their mental models evolved rapidly as they consume new information $[47,53]$. Contrastly, the simple temporally-ordered list of tabs allows users to access documents based on their open time. Yet as online tasks become increasingly complex and the number of tabs users keep around increases, recent anecdotal and empirical evidence suggests that this linear structure may no longer be sufficient [28, 28-30, 44, 57, 58]. For this, we focus our investigation on the challenges users face when trying to support modern online tasks using the simple linear structure of tabbed browsing. Based 
on the findings, we developed design implications that can inform the future development of web browser interfaces.

\subsection{Tabbed Browsing of the Web}

The way users access information online has gone through dramatic changes in the past 20 years. Most noticeably, online information seeking has evolved from navigating web directories (e.g., DMOZ [55]) for websites to searching for individual webpages and from supporting simple fact-finding tasks to exploring many webpages for complex sensemaking tasks [47, 54]. Meanwhile, an increasing proportion of desktop applications has also continued to migrate from the desktop to "the cloud" [15], such as communication, productivity, and entertainment. These changes reflect an increasing amount of dependence on modern web browsers' functionality and interfaces in meeting these needs.

Early work on tabbed browsing behavior mainly focused on how the introduction of tabbed browsing had gradually replaced the use of the back button as the primary navigation mechanism. Specifically, usage of the back button has seen a steady decrease from accounting for around $40 \%$ of in-browser navigation in the mid-90s $[6,64]$ to around $20 \%$ in the mid-2000s when tabbed browsing was first introduced $[73,74]$ and to $7.1 \%$ in the early 2010 s when tabbed browsing was supported by web browsers that had a combined market share of around 50\% [15]. For more in-depth studies on user behavior around tabbed browsing, Huang et al. [37] found that two-fifths of their participants prefer tabs for opening webpages in a search result in 2012, and Huang et al. [38] showed that at least $60 \%$ of peoples' tabs were related to other tabs of the same tasks in 2010. These findings provided evidence that browser tabs were used to facilitate complex sensemaking tasks that required users to consider multiple webpages in parallel. In a study closely related to this work, Dubroy et al. [15] interviewed 21 participants and reported rich qualitative insights into how browser tabs were used in 2010, such as revisitation and reminders.

However, the studies mentioned above were conducted around a decade ago when $50 \%$ of internet users did not have access to tabbed browsing functionality in their browsers [72]. In contrast, virtually all major web browsers support tabbed browsing today (August 2020 [72]). Additionally, most early work focused on ways users had benefited from the introduction of tabbed browsing compared to only using multiple windows, we instead focus on ways tabbed browsing can still be insufficient for complex tasks on the modern Web in order to inform future designs.

\section{METHODOLOGY}

This section describes a two-part study to investigate tabbed browsing behavior today. The first part was an in-person interview study that allowed us to discover deep qualitative insights around users' tab management strategies and challenges. The second part of the study supplemented insights uncovered by the interviews with an online survey to provide further empirical data and generalization. When describing our findings in the next section, we combine results from both studies to give a holistic view.

\subsection{Study 1: In-Person Interviews}

The first study aimed to investigate deep qualitative insights about the various functionalities browser tabs serve today and the potential breakdowns when conducting complex online sensemaking.

Participants: To maximize the richness of our findings, we used purposive sampling and targeted people who used a relatively large number of tabs to conduct complex online research. Specifically, we recruited participants across a research university and a research agency located in two states in the US for in-person interviews (California and Pennsylvania). We also pre-screened our participants using the following two criteria: 1) researchers who were actively working on one or more research projects or conducting literature reviews, and 2) people who self-reported often reaching 12 or more tabs open on their work computer. We continued to recruit at both sites while the research team members met regularly (often multiple times per week) to discuss interesting phenomena and trends in the data until new interviews consisted mostly of repeating ideas from previous interviews. In the end, a total of ten participants were recruited (Age: 19-32, $M=23.0, S D=4.7,40 \%$ male, mostly graduate and undergraduate students).

Procedure: Each participant was initially interviewed for 45 minutes followed by three additional 10-minute interviews over a two-week period. Each participant was compensated 15 US dollars for their time. Interviews were semi-structured starting with scripted questions and followed by open-ended discussion.

The initial interviews covered six topics including: asking about their active and recent tasks, probing about tabs that could be closed, their tab management strategies, and potential issues and emotions that arise when having too many tabs. Each topic started with one to six scripted questions (e.g., what would need to happen for you to feel that you could close this tab) followed by openended discussion. For example, we asked about participants' tab management strategies and their strengths and weaknesses with questions including "How frequently do you evaluate your tabs to see if you can close them? How difficult is it?", and "Does the number of tabs you have opened affect how you feel?"

Participants then were asked to walk through each of the browser tabs currently open in their work computers, explaining their tasks, goals, and purposes, why they were opened in the first place and the process of finding those webpages. We then further probed participants to discuss the reasons each tab was still open, using questions including "Was this tab intentionally kept around for later usage?" If answered "yes", we followed up with "Did you come back to it recently, why or why not?;" while if answered "no", we followed up with "Why was this tab kept around if you did not plan to use it again?" During the three follow up interviews over the next two weeks, each participant walked us through their open tabs following the same process as described above.

Analysis: The interviews were recorded, transcribed, and coded in four iterations following an open coding approach to identify common themes that captured rich qualitative insights grounded in data [3]. The analysis involved generating codes through two iterations of coding done independently by two authors who conducted the interviews. Subsequently, four authors had in-depth discussions to merge similar codes and form higher level themes. Finally, these consolidated codes and themes were applied to all transcripts to 


\begin{tabular}{lll}
\hline & \multicolumn{2}{c}{ Different Pressures for Closing versus Keeping Open Tabs } \\
\hline Close & C1: Limited Attention & Too many tabs makes it difficult to focus, overwhelming and causing stress \\
Close & C2: Limited Screen Real-estate & Too many tabs makes it difficult to navigate and have situational awareness \\
Close & C3: Limited Computing Power & Drains processing power causing browser and other applications to slow down \\
Close & C4: Pressure to be Organized & Social and self pressure to avoid looking disorganized \\
Keep & O1: Reminders and Resumption & Keeping tabs around as a reminder to work on them or keep track of progress \\
Keep & O2: Revisiting References & Keeping frequently used tabs for quick access; has a diminishing return \\
Keep & O3: Avoid Costly Re-finding & Avoid closing tabs in fear of missing out on valuable information \\
Keep & O4: Sunk Costs and Aspirations & The hopes to process more info than capable; while aware of the situation \\
Keep & O5: External Mental Model & Memory and mental model; Organize tasks with windows, desktops, browsers \\
Keep & O6: Uncertain Relevance & Difficulties in judging the current and potential relevance of tabs in the future \\
\hline
\end{tabular}

Table 1: An overview of our findings from Study 1: Two sets of opposing pressures that drive tabbed browsing behavior.

arrive at the findings in the paper We focused our analysis on investigating the issues and challenges our participants faced when conducting research online, and we converged on themes that could be grouped into two sets of opposing pressures for keeping tabs open (six categories) and closing them (four categories) as shown in Table 1). These categories are described in detail in the following section.

\subsection{Study 2: Survey with Mechanical Turk}

While the interviews provided deep qualitative insights into the different breakdowns of tabbed browsing today, the second study aimed to provide further context and frequency information around these challenges with the broader population. For this, we conducted an online survey with 103 participants recruited from Amazon Mechanical Turk (Age: $21-70 ; M=36.33 ; S D=10.65 ; 49.5 \%$ female; mostly from the US) [41]. While prior work has also used crowdworkers to understand browser usage behavior, but focused only on how they managed crowdsourcing related tabs [76]. Here, we explicitly asked our participants to only include their tabs that were unrelated to crowdsourcing tasks in their responses to get a better picture of their more general browsing behavior.

In contrast to participants in the first study that were graduate and undergraduate researchers, participants in the second study covered a much wider variety of backgrounds. Based on selfreported data, the most common occupations were software and hardware engineers (13\%), management (10\%), retail and customer service $(8 \%)$, IT $(8 \%)$, analysts $(6 \%)$, and healthcare providers $(5 \%)$. The most common education backgrounds were Bachelor's degree (53\%), high school degree or equivalent (24\%), and Master's degree (19\%). Each task took participants around 20 minutes to finish, and each participant was compensated with 3 US dollars for their time.

The main part of the survey consisted of two parts. The first part focused on their general experiences with tab management, such as how often they feel overwhelmed by the number of open tabs, or what were their thresholds for the number of open tabs. The second part focused on their experiences with being pressured to keep tabs open. For this, participants gave a short description for each of their open browser tabs at the time when the survey took place (excluding tabs related to Mechanical Turk tasks, such as our survey page). Participants then further reported why each tab was kept open by assigning different pressure types for keeping tabs opened as uncovered by Study 1 (Table 1, more details in the next section). To control for survey duration, participants reported up to 10 browser tabs if they had more than 10 , which accounted for $7.8 \%$ of all participants $(\mathrm{N}=103)$. On average, each participant labeled 6.15 tabs ( $\mathrm{SD}=3.69)$, or a total of 633 tabs. Each tab was assigned with an average of $1.39(\mathrm{SD}=0.92)$ of the six pressure types, or a total of 880 labels.

\section{PRESSURES TO CLOSE VS. KEEP TABS}

Overall, we found that people had mixed feelings about their tabs. When they have a manageable number of browser tabs, participants felt in-control and productive about using them. This positive view is consistent with prior work, including a study on Firefox users in 2010 [15] that found positive perceptions of using browser tabs when compared to only using browser windows. However, unlike the prior study, all participants also described negative emotions and pressure when the number of tabs became unmanageable. These findings are more consistent with results from more recent work on "hoarding" of digital files [71]. Even though tabs were considered more ephemeral than files, our participants expressed feeling attached to the information saved in tabs and invested in the organization they built up, making it difficult for them to reduce the number of open tabs. We posit that these forces represent two fundamentally opposite pressures - to close tabs vs. to keep them open - that lead to challenges for users and opportunities for new interface paradigms to explore. Table 1 shows an overview of our findings, and below, we discuss these two opposing forces that govern users' tab behavior based on evidence from both the interview data from Study 1 and survey results from Study 2.

\section{PRESSURES TO CLOSE TABS}

On the surface, the linear structure of browser tabs allows users to open, retain, and manage an unlimited number of webpages. However, participants expressed several negative feelings when they accumulated too many browser tabs and described the reasons behind them. We argue that there are, in fact, different implicit costs associated with creating new tabs to load more webpages, and that browser tabs should be considered a limited resource. To better understand this limitation, we asked participants in the survey 
about the number of tabs they would start feeling overwhelmed and experiencing difficulty managing them. The responses followed an long-tailed distribution showing that people have different tolerances (Figure 1), with the median number being eight tabs $(\mathrm{N}=103$, first quartile: 5 tabs; third quartile: 12 tabs). We further asked them how frequently they reach this threshold. The majority $(67 \%)$ of our participants responded at least once a week (17\% daily; 9\% 4-6 times per week; $25 \%$ 2-3 times per week; and $16 \%$ once per week), showing that tab management has now become a common issue for users. From analyzing the interview data for deeper qualitative insights, four common themes emerged: tabs incurring pressure on attention (C1), tabs taking up screen real-estate (C2) and computing resources $(\mathrm{C} 3)$, and people feeling a desire to appear organized to themselves or others (C4). Below we discuss these in more depth.

\section{1 [C1] Pressure on Attention}

A fundamental problem is people's limited attention. In situations where people are faced with too many options and decisions to make, it becomes harder to focus on what is important $[60,77]$. Similarly, having opened too many tabs can potentially cause users to lose track of all the information sources they have on hand and lose focus on which tabs they should concentrate on. Participants we interviewed in Study 1 expressed how they were often overwhelmed by the large number of tabs they have opened and how it made them unable to focus on the important tasks.

"I think probably, overwhelming is a good word. It's not like it's helping, it's not like I can suddenly find things better when I have that many tabs open, because that's definitely not the case. So, I think the more tabs I have open then it's more of an indication that I'm in the middle of a mix, and I just don't have time for, like, basic human cleaning functions." Participant J3

Conversely, participants also pointed to how closing and reducing the number of tabs can relieve pressure and stress caused by their browser tabs.

"Usually, it's a relief [to be able to close browser tabs], even if I'm missing things that I think would have been important. I can't usually remember specifically what they would have been... When I am able to close a [browser] window I also feel like that's great." Participant J2

\section{2 [C2\&C3] Pressure on Screen Real-estate and Computing Resources}

Besides limited attention, many participants also mentioned screen real-estate as another limited resource. They expressed how having too many tabs leads to difficulties in navigating and finding previously opened tabs. More specifically, creating each additional tab reduces the width of existing tabs causing a smaller portion of the webpage titles to be rendered by the browser. Further, they pointed to a breaking point for tabbed browsing: When browser tabs became so narrow that the favicons were no longer rendered, tabbed browsing became virtually unusable.

\begin{abstract}
"When I can't see the little icon. Then that's too many... I can otherwise definitely find what I want with like 3 clicks at most, generally 1 click. It's once I can't see the little icons..." - Participant $\mathrm{H} 4$

“As for like a mental burden...It's crazy. This doesn't happen very often, but if I have enough that the titles or icons don't show, I'll be like, "Wow, how did I get this bad?" And get really bothered by it" - Participant $\mathrm{H} 2$
\end{abstract}

In extreme cases, participants who sometimes keep a large number of tabs around mentioned having too many tabs drains the limited processing power and memory space from their computers, causing their browsers to become too slow.

"I'll try to go into different browsers to see if there are tabs that I just don't need. so, yeah, usually the only time that I do that is if Chrome is starting to get really slow." - Participant J2

One participant in the interview study, J3, even described how it inhibited him or her from using other desktop applications normally.

"But right now [at the time of the interview], my battery drains so fast because I have all these tabs open, that it actually inhibits a lot of things I'm doing." - Participant J3

These qualitative insights further confirmed results from the preliminary survey (described in the Introduction), where $28 \%$ of the participants $(\mathrm{N}=64)$ agreed that they often struggle with finding the tabs they needed in their browser, and 25\% reported having experiences with browser or computer crashing in the past due to having too many browser tabs opened in the past.

\section{3 [C4] The Pressure to be Organized}

As described above, depleting the different limited resources created significant issues for our participants trying to manage all their open browser tabs. Indeed, many participants described how browser tabs could become a source of stress and frustration due to these limitations, especially when they try to navigate and manage many tabs in parallel.

“... but if it seems like I have a lot of tabs open, I try and go through and see which ones I can close. But sometimes, that's very frustrating because sometimes it's like I can't exactly close any of these right now, but there are so many of them" - Participant H1

More interestingly, some even noted feelings of shamefulness for having a large number of tabs opened, and their unwillingness to reveal their tabs to others.

"Because it's...shameful in a way. It makes, like I feel like I would have been giving, like a bad impression to these people that I, that had to look at my screen." Participant J3

As a result, some participants may even decide to "clean up" their tabs before they have to share their screens with others:

"I'm going to do a presentation and I care about what people are going to think about me when I plug my 
If I have more than _ tabs, I would start having issues managing them.

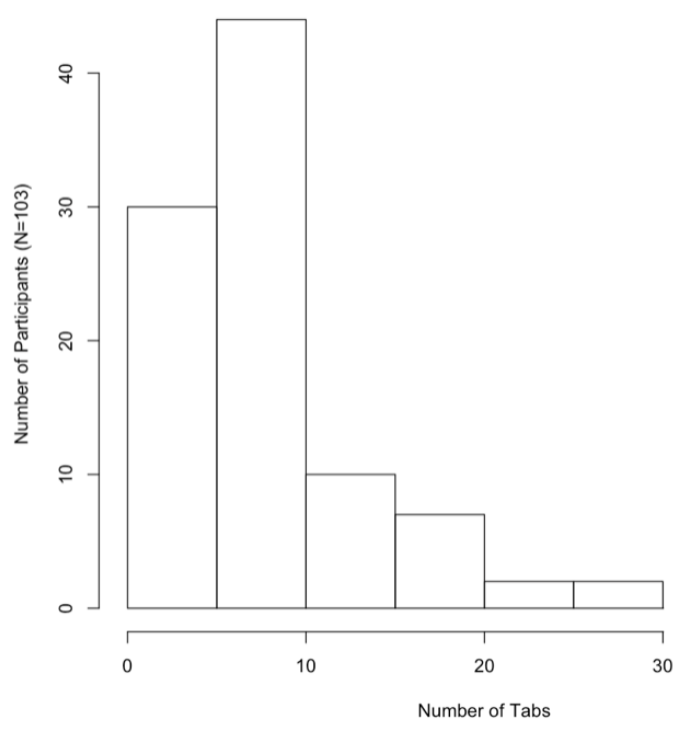

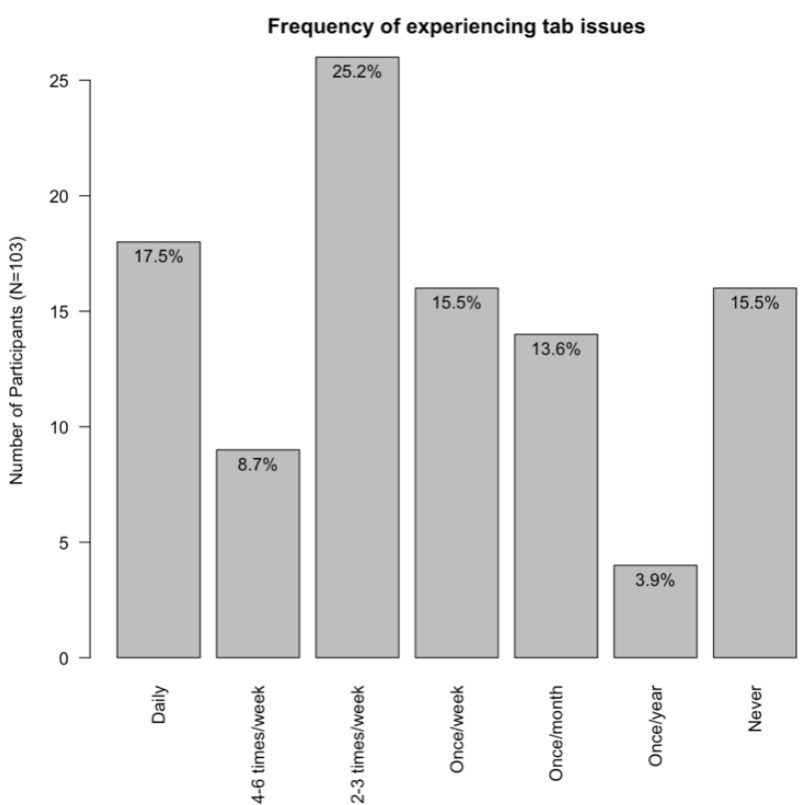

Figure 1: When asked about having how many open tabs they would start experiencing issues managing them, responses from the survey participants in Study $2(\mathrm{~N}=103)$ followed a long-tailed distribution with the median number being 8 tabs $($ first quartile: 5 tabs; third quartile: 12 tabs). When further asked about frequently they reach their thresholds. The majority (67\%) of our participants reported at least once a week.

computer in, then that's when I might clean it up" Participant J2

These results suggest that users are, in fact, motivated to maintain a clean and organized workspace, but were often failing to do so while conducting tabbed browsing. This could potentially demotivate users to collaborate in-person with their co-workers in an office setting or even remotely using screen-casting tools [2].

\section{PRESSURES TO KEEP TABS OPEN}

Given the above pressures caused by having too many tabs, and the seemingly low cost of closing tabs to relieve such pressure, why do participants still frequently find themselves in situations where they "hoard" too many tabs? Close examination of the interview transcripts revealed six common pressures for why people open and keep a large number of tabs as shown in Table 1, many of which were dependent on the usefulness and relevance of a tab over time, leading to issues such as keeping around obsolete tabs that could have been safely closed. Using the survey responses from Study 2 , where participants labeled their open tabs with the six pressure categories, we also present how common these pressures occur at a larger scale.

\section{1 [01] Reminders and Unfinished Tasks}

All participants in Study 1 pointed to tabs that were intentionally kept around as reminders for unfinished tasks.
“And sometimes, when you're in a rush, you don't really have time to focus on which tabs you really need, so you'll put it off for later." - Participant H2

"Yeah it sort serves not in a "Oh shit" way but in more of a, sitting there nagging me like a mother sort of way. And I would say it's effective in doing that." Participant J3

This usage is consistent with prior work that focused on browser tab usage during search sessions [37] and general tabbed browsing [15]. Our survey results from Study 2 further showed that this is a common reason for keeping tabs around with $37.3 \%$ of the browser tabs being labeled as reminder tabs $(\mathrm{N}=633$, Figure 2).

Since participants mentioned that these reminder tabs are often inactive for extended periods, one currently available solution could be to save them as bookmarks so that tab resources can be released. However, when prompted, participants expressed how bookmarks do not exhibit the same reminding functionality, citing the high cost of creating and accessing bookmarks and that once created, they are "out of sight out of mind".

"Yeah, I would say bookmarks for me feel like a deep archive... when you bookmark something... you just kind of puts it all in a pile" - Participant J5

"If I bookmark everything as default [without a category], it would just get added to a long list of longforgotten sites that I bookmarked randomly... I want to bookmark it and put it into a category but then that requires figuring out what category to put it in, 


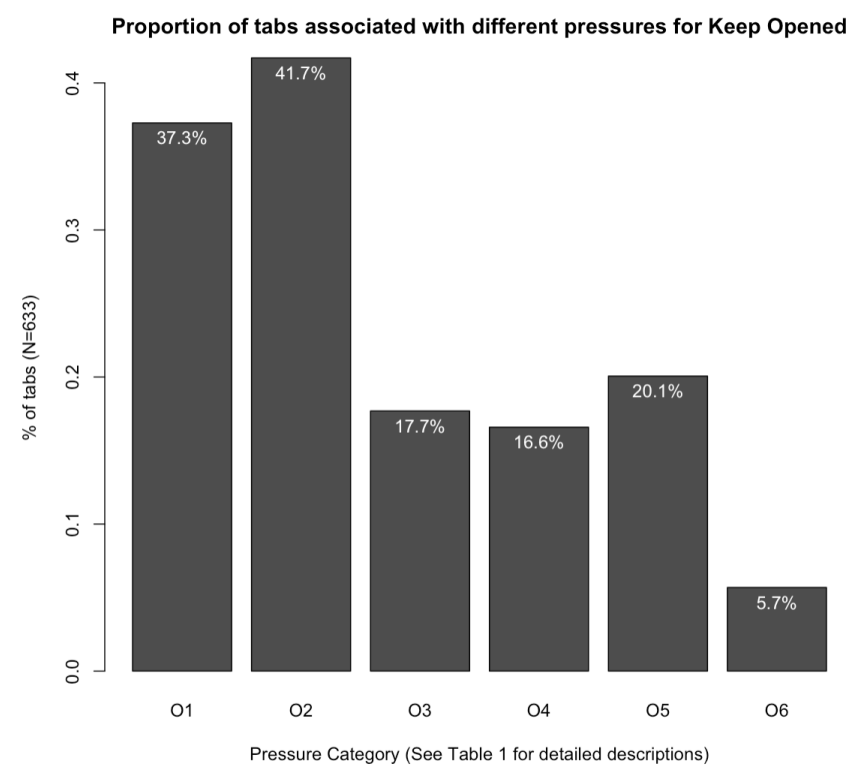

Figure 2: Participants $(\mathrm{N}=103)$ in Study 2 labeled their open tabs $(\mathrm{N}=633)$ with different pressures identified in Study 1. This figure shows the proportion of tabs associated with each pressure types. Each tab can be associated with more than 1 pressure types.

which as you know, requires a lot of cognitive load." Participant J1

While participants seemed to agree that creating structures, such as categories or nested folders, for saving tabs into bookmarks brings benefits such as ease of access and a better understanding of the information landscape [62], high cognitive costs of categorizing browser tabs seemed to be prohibitively high [45]. Prior work on online information foraging showed that users often do not have sufficient understanding of the information space to create effective structure, which provides a potential explanation as to why saving tabs under categories can be costly to users. More fundamentally, bookmarks do not provide reminding functionalities to our participants. Specifically, saving browser tabs as bookmarks and closing them causes tabs to lose their ability to "nag" the users from time to time.

\section{2 [02] Revisiting Frequently Accessed Pages}

The most common functionality of browser tabs is to reduce the cost of repeated navigating to frequently revisited webpages. Results from Study 2 showed that $41.7 \%$ of our participants' browser tabs were references that they frequently accessed $(\mathrm{N}=633$, Figure 2$)$. Participants from the interview revealed these references to be a mix of single-page applications, such as email clients and calendars and information references supporting larger projects that consists of multiple browser tabs.
"I usually try to have one window of my core stuff, so my window right now has my email, calendar, and capstone project stuff that I can reference really quickly." - Participant J2

However, immediately afterward, J2 noted that the benefit of keeping frequently accessed tabs around has a diminishing return: as the total number of tabs increases, users' ability to efficiently access specific tabs decreases. J2 summarized this cost structure balance with an apt analogy to putting clothes away:

"For me it feels like other things that I do that don't make a lot of sense, but feel like the right things to do. So I've often have a chair in my room that I'll just drape clothes back over it instead of putting them away. Because if it's a sweater that I wear a few times a week, it'll be easier if I just get that sweater that's draped over this chair than if I put it in my closet. But then that becomes like 30 things draped over the chair, and I actually can't find anything draped over the chair. I know it doesn't make sense but I still do it. Like, in the moment it seems like the right thing to do, the easier thing to do." - Participant J2

This tension leaves users the heavy burden of carefully balancing between the number of frequently used tabs to keep around and how efficiently those tabs can actually be accessed.

\section{3 [03] Avoiding Costly Re-finding}

Besides keeping tabs open to reduce revisitation costs, participants also noted how closing tabs can potentially incur the high costs of refinding them if they become relevant again in the future. Although less common than the first two pressures, Study 2 showed that a significant portion of browser tabs were kept open to avoid this potential cost at the time when the survey took place $(17.7 \%, \mathrm{~N}=633$, Figure 2).

On the one hand, participants described strategies they use for re-finding previously closed tabs, many were consistent with findings in the literature, including retracing the small steps they took in the first place to find them [67] or reissuing the same queries to a search engine [66]. On the other hand, participants still expressed their fear of closing the wrong tabs, either by mistake or by misjudging their relevance, and pointed to cases where they had to pay the price of re-finding valuable tabs. One participant even stated that he or she would go through history to find valuable tabs that were closed, while others were willing to lose information they considered valuable to avoid having too many tabs.

"But in all likelihood, there's been a few tabs where I've saved and I've never come back to. Or it gets closed out accidentally. Which is a very scary... A fear of mine where a tab will get closed out and I won't know what I'm missing... It's the fear of missing something important or something that will lead to enlightenment, to more knowledge, or something that will help you get a job. It's the fear of missing out." Participant J1

"I would actually go through my browser history and go "I had this tab open, I had this tab open, I had this 
tab open" and then I would reopen a lot of those tabs." - Participant J4

One scenario commonly described with the high cost of re-finding tabs was during online collaboration:

"[I kept this tab open] because I'm not confident I know how to get back to it. Especially if it was a link from Slack ${ }^{1}$ or someone else created in our [shared] drive... For the files that I've created, I know where they are so I know where to find them. But in the case of other people's files, I don't know where they are so I am more likely to keep tabs open for files that other people create because I don't know where they filed it." - Participant J1

These provided an example scenario where the pressure for keeping a tab open increases with the higher difficulty of re-finding it.

\section{4 [04] "Irrational" Pressures: Sunk Costs and the Aspirational Self}

Participants also cited reasons for keeping tabs around that may not fit in a rational economic analysis view of the cost structures of tab usage. Two common patterns were the sunk cost of creating and managing tabs and the disparity between a participant's actual and aspirational self.

Sunk Cost. When participants spent efforts to find and organize their tabs, they felt invested and that tabs had inherent value to them beyond efficient navigation. This reluctance to close tabs may be a form of loss aversion [68], in which simply the "ownership" of a tab may give it value:

"It made me think about how it's weird that even when I'm not using those tabs, I don't want to close them. Maybe it's because it took efforts to open those tabs and organize them in that way." - Participant H2

Aspirational Self. Another form of "irrational" behavior reminiscent of loss aversion we found was the mismatch between a participant's goals for their aspirational self and what would be realistic for their actual self. Specifically, the amount of information participants planned out to consume can sometimes exceed their available time and attention. This discrepancy between the ideal state and the actual state of self can cause negative emotions, such as dissatisfaction and disappointment [35].

One reason is that for many tasks there is virtually unlimited relevant information given the scale of the modern Web. Users with a maximization tendency may spend vast amounts of time trying to collect all relevant information to avoid the chance of missing valuable insights [61]. Furthermore, users often encountered and collect various interesting articles or websites in their social media or news feeds that they would like to read someday. Indeed, participants reported that sometimes the amount of information they hoped to process exceeds their capability and resources.

"It kind of becomes this kind of mess that you see here [referring to tabs of unread article], just a big list of tabs that I was supposed to get back to but never did." - Participant J4

\footnotetext{
${ }^{1}$ Slack. An instant messaging application. http://www.slack.com
}

"It's not really worth it, but at the same time, I don't want to be like "Oh, okay, that's it. I'll never remember [to read these articles]." So I just leave them there, and I might never come back to it" - Participant H4

"To be honest, it's more like I will collect a bunch of links and then never really ever go back to them. When I figured this out, I sort of stopped caring about bookmarks. Sometimes I will throw things into this other bookmark [referring to the bookmark folder] that's more unorganized, but I don't think I ever look back on these." - Participant J4

Interestingly, when asked about their own expectation for eventually processing them, participants admitted that realistically they would never actually get to them. This suggests that users are aware of the disconnect between the amount of information they aspire to consume versus their capacity to process all their open tabs. This pressure also accounted for a surprisingly high proportion of participants' browser tabs from Study 2, with participants reporting that, on average, they might not ever process $16.6 \%$ of their open tabs $(\mathrm{N}=633$, Figure 2).

\section{5 [05] External Mental Model}

Participants expressed how browser tabs act as a manifestation of their mental models, using them as an external - yet transient memory store in a similar fashion as an active working memory [1]. Based on survey data, this category corresponds to one fifth $(20.1 \%)$ of all tabs $(\mathrm{N}=633$, Figure 2) reported by participants in Study $2(\mathrm{~N}=103)$. Interview data from Study 1 further showed that participants maintained sets of tabs corresponding to their external model for larger projects:

"It's like a manifestation of everything that's on my mind right now. Or the things that should be on my mind right now... So right now, in this browser window, I have a web project that I'm working on. I don't have time to work on it right now, but I know I need to work on it. So it's sitting there reminding me that I need to work on it." - Participant J3

Our participants typically conducted a wide range of different tasks in parallel or interleaved, sometimes engaging in multiple productivity and leisure tasks simultaneously.

"I just opened it in Firefox and I had Hulu and Netflix open there [in one window] and it felt like it was good to keep it separate from my more serious work related tasks so I wouldn't get distracted if I'm in like a this one [a separate window]... I wouldn't see the little Netflix icon and get tempted." - Participant J2

The simple linear structure of tabs was often insufficient for organizing multiple complex tasks simultaneously, and participants had to resort to various strategies and tools to create more sophisticated structures to better represent their mental models.

"I would usually try to keep either different windows or even different browsers like Chrome for work and Firefox at home so that I wouldn't mix those things up." - Participant J2 


\begin{aligned} & \hline Related Pressures Design Probes based on Existing Systems \\ & \hline O4, O5 D1: Archiving tabs into sessions (similar to OneTab [19] and SessionBuddy [23]) \\ & O4 D3: Grouping tabs into workspaces, (similar to Workona [20] and Toby [24]) \\ & O2, O5 D4: Searching for keywords across tabs (similar to Search all Tabs [22]) \\ & O1, O6 D5: Prioritizing tabs similar to to-do items (similar to Todoist [39]) \\ & \hline Related Pressures Design Probes based on New Designs \\ & O1, O4 D6: Allowing users to "snooze" tabs and be reminded in different ways in the future \\ & O2, O3 D7: Preserving the context of open tabs using visual indicators (e.g., progress, time spent, and provenance) \\ & O2, O3 D8: Same as above but for recently closed tabs \\ & O5 D9: Automatically clustering tabs into nested folders based on seed suggestions provided by the user \\ & O5 D10: An always-present sidebar for saving and organizing pages, clips, and notes \\ & \hline\end{aligned}

Table 2: Design probes used in the exploratory interviews for developing design implications.

\begin{abstract}
"I don't like it when it gets so tiny you can no longer see the little favicon. So usually I would group [tabs] thematically. Like if I have a bunch of job search tabs open, I would have them all in one window. If I'm working on a web project, I would have those in another window." - Participant J3
\end{abstract}

While the strategy of using multiple browser windows to create two-level hierarchies of tabs was consistent with a study conducted ten years ago [15], our participants also reported utilizing operating system features to create task hierarchies, such as using virtual desktops and even multiple browsers or computers (work computers versus personal computers) to create more levels of hierarchies or to separate task contexts.

\section{6 [06] Uncertain and Changing Relevance}

In the preliminary survey (described in the Introduction), only $19 \%$ of the participants $(\mathrm{N}=64)$ agreed that laziness contributes to having browser tabs that were no longer useful. Participants we interviewed in Study 1 shed light on possible alternative explanations centered around the difficulties of estimating the expected value of tabs. Specifically, it can be challenging to determine the relevance of a tab for current and future tasks. This is especially true when the task may not have one correct answer, the answers found do not perfectly suit the user's goals, or that the user is too early in the process to be confident in an answer even when first seen:

"The next three tabs are all different responses for that [referring to a problem at hand]. Because none of them seem completely wrong, but they also don't seem like exactly what I'm looking for [so they were currently kept open]" - Participant H1

As a result, users can be tempted to keep all potentially relevant tabs open until confident that they would no longer be needed. Prior work has suggested that some users may have difficulties deciding how much information they should copy and save during online exploration [7]. Our participants directly reported that the relevance of opened tabs might change over time as they learned new information, which potentially explains why relevance judgment can be difficult.
"A lot of these [tabs] I opened in the beginning [of a task]. I forgot that I even had them. This was a concept that I thought I would start using, but then I ended up using something else." - Participant $\mathrm{H} 3$

"Sometimes it feels like that moment has passed or for whatever reason making the others [referring to a group of tabs] didn't really feel relevant anymore." - Participant J3

Fundamentally, when learning new information online, it can be challenging to judge the relevance of a tab because the users have not yet sufficiently explored the information space. However, learning new information can invalidate multiple existing tabs, but the cost of locating and closing them can be prohibitively high. While this only accounted for $5.7 \%$ of the tabs reported in Study 2 $(\mathrm{N}=633$, Figure 2), interview data from Study 1 suggests that this could be a fundamental cause for quickly accumulating tabs that no longer had values to the users, especially for information workers conducting complex online research.

\section{IMPLICATIONS FOR DESIGN AND EXPLORATORY INTERVIEWS}

To help further develop our user model into a set of design implications, we conducted an exploratory interview focused on potential solutions with seven additional participants (age: one 19-24, three 25-34, one 35-40, one 41-54, one 55+; four female, three male). Participants were all working professionals recruited through posts on social media, ${ }^{2}$ and each was interviewed for 60 minutes and compensated with 50 US dollars for their time. Based on our user model, we generated ten design probes prior to the interviews that aimed to test the drivers underlying different tab management pressures. Ten design probes were used in the interviews as listed in Table 2. Five probes involved functionality found in existing tools, and five involved possible future functionality. These probes helped us explore the design space of possible tab management approaches with our participants, and identify potential future designs addressing their individual limitations.

\footnotetext{
${ }^{2}$ Facebook, LinkedIn, and NextDoor
} 


\begin{tabular}{|c|c|c|c|}
\hline Section & Design Implication & Pressures & Summary \\
\hline 7.1 & Task-Centric Context Switching & $\mathrm{C} 4, \mathrm{O} 5$ & $\begin{array}{l}\text { Browser interfaces need better ways to compartmentalize tabs by users' } \\
\text { task contexts and structures so that users switch between different tasks } \\
\text { and subtasks more easily. }\end{array}$ \\
\hline 7.2 & Task Mental Models & $\mathrm{C} 1, \mathrm{O} 5, \mathrm{O} 6$ & $\begin{array}{l}\text { Browser interfaces should provide richer structures that can better } \\
\text { reflect users' mental models, making it easier for them to orient them- } \\
\text { selves in the information space and resume prior tasks. }\end{array}$ \\
\hline 7.3 & User Attention Management & $\begin{array}{l}\mathrm{C} 1-\mathrm{C} 3, \\
\mathrm{O} 1-\mathrm{O} 4, \mathrm{O} 6\end{array}$ & $\begin{array}{l}\text { Browser interface should help users better manage their attentions, } \\
\text { dynamically changing tabs salience to reflect their current relevance, } \\
\text { making it easier for users to focus on what is important. }\end{array}$ \\
\hline
\end{tabular}

Table 3: An overview of the design implications.

During the interviews, we first walked through each participant's online research process, probing their use of tabs, bookmarks, and other productivity tools, and explored challenges, pain points, successes, and ideal states. Doing so allowed us to better understand the tasks and challenges familiar to each participant so that we could present our designs in relatable scenarios. We then walked through each of the ten designs and used them as probes to investigate participants' attitudes towards solutions addressing different tab management issues and their threshold for introducing new functionality into their existing workflow. Finally, combining findings from Study 1, Study 2, and the exploratory interviews, we proposed three main areas below where the browser interface can be improved to relieve current tab pressures. Table 3 shows an overview of our design implications and the pressures they aim to address.

\subsection{Task-Centric Context Switching}

Our participants organized their attention at the task level and often needed to switch between sets of tabs supporting different tasks. They saw sets of webpages as external representations of their mental models for different tasks. This suggests that future browser interfaces needed to provide better compartmentalization of tabs at the task level. While commercial solutions such as Firefox Containers [75] or using multiple accounts in Chrome allow users to separate their tabs for different high-level contexts (e.g., work vs. personal tabs), our participants often had large numbers of smaller tasks under each context, for example, researching restaurants and activities in different parts of a new city when planning a trip.

One issue with the current uniform order and spacing of browser tabs is that it can be difficult to see clear task boundaries when working on multiple tasks in parallel. Furthermore, when a user switches back and forth between tasks, newly created tabs for different tasks can become interleaved. As a result, the more often users switches between tasks, the more fragmented their tabs can potentially become, making it increasingly challenging to switch context between tasks. Some of our participants ameliorated this problem by using different browser windows for different tasks. This same strategy is also seen in commercial browser extensions such as Workona [20]. However, participants also described issues with not being diligent enough to always switch to the correct project window before creating new tabs, and that even misplacing a few tabs in the wrong window can have adverse consequences, for example, difficulty when trying to re-find a tab and missing out on important information buried in the wrong task window. Fundamentally, designs that require users to put in extra effort may work well in scenarios where they have relatively static sets of tabs they wish to "bookmark" into groups. However, for exploratory scenarios involving frequent opening and closing of many tabs, the amount of effort required to keep tabs organized may be prohibitively high. Addressing this in future designs could greatly reduce tab pressure when users conduct multiple tasks in parallel.

One potential design space is to make task context more explicit and raise awareness of which task context is currently active (for example using visual cues to show task boundaries). An upcoming update to the Chrome Browser interface (in a gradual release as of September 2020 [69]) included a design where users could create named sections of tabs within the same window and color-code them, making task boundaries more visually salient. However, it still required significant user effort to put tabs into the right sections. Specifically, even when users are currently in a specific section, newly created tabs are still put under an uncategorized section. While this could potentially reduce the chance of users misplacing tabs into the wrong section, it also required users to put in significant manual effort, for example, manually assigning tabs from new searches into the right sections.

Another potential direction is for browsers to proactively lower the cost of organizing tabs by tasks by computationally identifying latent task topics and automatically suggesting existing workspaces for newly created tabs. Participants from the exploratory interviews responded positively to this design idea, explicitly citing how $a$ smart grouping of my tabs supporting expanding and contracting by task would be useful allowing them to jump in and out of different clusters of tabs. At the same time, some raised concerns about the accuracy of such predictions, stating that it would only be useful if the automatic predictions were accurate enough. Search engines have long been able to group sets of queries into larger search tasks with high accuracy [40,63]; perhaps these techniques could also be adapted to predict user intentions when a new webpage is loaded and assign (or reassign) its tab to the right task context.

In sum, a fundamental challenge here for interface designers is that when users create new tabs, their intentions could be either to continue the same task, to context switch to another existing 
task, or to start a new task. Making an assumption to use as default behavior could lead to users misplacing their tabs in the wrong task context and incur high costs for recovery. Addressing this future design, through user interaction and/or computation, has the potential of greatly reducing tab clutter by lowering the costs of keeping tabs organized by tasks. Many of the pieces for realizing this are available today. For example, several signals are available for detecting user tasks that have been used in backend web services, such as temporal search query segmentation [40] and semantic clustering [43]. Web browsers also offer the potential for exploiting user interaction signals in the frontend (e.g., tab switches, dwell time). Tasks could be inferred from these signals using binary classification (e.g., whether tabs are in the same or different tasks) or using clustering algorithms (e.g., hierarchical approaches which might infer sub-tasks). A key challenge with realizing this approach is recovering from errors: since models are unlikely to reach $100 \%$ accuracy, it will be important to develop interaction paradigms in which users can fix errors in a lightweight way, ideally with the system using these fixes to improve itself over time.

\subsection{Task Mental Models}

Users need better support for creating rich structures when conducting complex tasks in the browser. Participants from Study 1 described that many of the pressures in our user model frequently occurred when they were conducting complex tasks involving many tabs. They described these tabs as external mental models for keeping track of their research progress, and external memories that hold different reminders for tasks they wish to complete. Unsurprisingly, the linear design of browser tabs often can not fully capture these complex mental models. In this case, some users resort to creating separate documents to save URLs, gather information across webpages, and keep track of their ideas (e.g., word documents or spreadsheets). This suggests that it could be beneficial for browsers to provide integrated support for conducting complex tasks with more sophisticated structures that can better reflect users' mental models.

Tabs should better reflect users' complex task structures. One potential design space is to bootstrap such mental model representations with minimal user effort by identifying their intentions using their navigation patterns. For example, a set of tabs opened from a search engine query is likely to support the same information needs; or, a set of tabs opened from a top-10 list article are likely competing options under the same category. Capturing and organizing tabs using such structures has the potential of better orienting users and providing better support for task progression and resumption. Additionally, it also has the potential of addressing accumulating tabs that were no longer useful due to their changing relevance over time. For example, once a user decided which city to visit early on in a travel planning task, such structures could allow her to quickly close sets of tabs for researching other cities that she had considered. Prior work has shown promising results for grouping tabs opened from the same searches to better support users in conducting exploratory search tasks on desktop [49] and on mobile devices [32]. Ways to generalize this approach to support other scenarios can be a fruitful design space.
Allow users to externalize their thoughts and synthesize information across tabs. As the browser increasingly becomes the primary tool for supporting research and productivity, another area that lacks native support is to allow users to externalize their thoughts and synthesize content across webpages. Participants in both Study 1 and the exploratory interviews both pointed to scenarios where tabs were only a small part of a collection of notes, lists, files, etc for a task, and pointed to how they used external word documents to take notes, copy and paste content from tabs, and to organize relevant URLs. Much literature has pointed to the importance of synthesizing pieces of information across sources when users conduct online research across different decision-making scenarios $[12,27,56]$, and that articles synthesized from multiple online sources by crowdworkers were preferred over individual top search results [10,31]. More directly, a recent survey showed that around half of their participants $(49.4 \%, \mathrm{~N}=89)$ use spreadsheets to gather evidence and take notes across multiple online information sources to compare options (e.g., products or destinations) to help them make decisions [8]. However, current browsers treat tabs as individual silos and provide little support for cross-referencing and collecting information between webpages. Using external tools, such as word documents and spreadsheets, creates a disconnect in users' workspace, and can incur high cognitive and interaction costs when trying to copy and paste information to synthesize them in a separate document [8].

Recent commercial and research systems have started to address this issue. For example, the Collections feature in the Edge Browser allows users to make lists of images, texts, and links collected across different webpages; research systems such as Mesh [8] and Unakite [46] are also promising examples of tightly integrating note-taking and online exploration in the browser to capture user mental models. For example, Mesh allows its users to extract reviews for different products and criteria to build up comparison tables and allows users to make personalized judgments to keep track of their progress, leading to users learning facts about products more efficiently and gaining deeper qualitative insights from the reviews [8]. Readily available NLP techniques that provide a deeper semantic understanding of documents, such as linking entities to ontologies [48] and word-semantic models [14, 52], can further reduce the interaction costs of such systems by computationally identifying relevant content across webpages in the browser. Latency and accuracy will be important in the viability of such approaches.

In sum, one fundamental reason users conduct parallel browsing is to make sense of the larger information space, and the above evidence points to a strong need for tools that can better support synthesizing evidence scattered across webpages into flexible structures (i.e., beyond lists and tables) and to support a broader range of online tasks (i.e., beyond product comparison). Realizing this could allow the browser to evolve into a more comprehensive and integrated research support tool.

\subsection{User Attention Management}

Users have limited attention and capacity to manage and navigate across many tabs and windows. Future systems need better ways to help users focus their attention on important tabs while avoiding distractions from tabs that were not relevant to their current 
tasks. Even within one window, as the number of tabs increases, it becomes more challenging for users to find relevant information or to identify which tabs they should focus on. One participant described how difficult it was for them to find what they needed when they had many tabs open: "I hate having a million tabs. I really try to close the tabs I know I won't need. I know it's too much when I can't find what I'm looking for." One reason is that tabs are currently designed to have equal salience (e.g., same tab-width) and always present to draw the same amount of attention from users regardless of importance or relevance to their current task. Below we discuss design implications for future interfaces that can help users better focus their attention on tabs that are important to their evolving goals, contexts, and interests.

Reducing the salience of aspirational and ToDo tabs. Future systems need to reduce the salience of less important tabs to reflect how users prioritize them. Participants in our study described accumulating tabs around for reminding them of future tasks and to collect articles they want to read, but struggled to prioritize them causing stress and clutter in their browsers. For example, one participant said how having many tabs could be stressful as the tabs were all treated as incomplete tasks: "It stresses me out to have so many tabs open. It's like a ToDo list with lots of things that aren't done." Conversely, when a user is engaging in a productivity task, such as writing a paper, finding important tabs also becomes more challenging (e.g., tabs for document editing or references) when there are many other less relevant tabs (e.g., emails to reply, news articles to read, or social media). This illustrates how existing tab designs lack support for prioritization, and highlights opportunities for helping users focus on tabs currently important. One current solution to reduce the salience of less important tabs is allowing users to "put them away" by bookmarking or archiving them. However, this could also cause issues with resurfacing and reaccessing them as described below.

Avoid black-hole effect. Participants mentioned that the existing design of bookmarks lacked reminding functionalities and worried that they might never get back to tabs once they were out of sight. The high cost of re-finding tabs further exacerbates this "black-hole" effect once tabs are closed or archived. While creating structures over tabs for bookmarking may lower the cost of re-finding them, the cost of categorization can be too high for many users [45]. As a result, participants resort to archiving tabs without any categorization or structures. For example, existing tools like OneTab $[16,17,19,23]$ allow users to archive and restore multiple tabs, but the lack of mechanisms to resurface them can lead to large numbers of tabs being archived and never re-accessed. To address this issue, future systems may proactively resurface archived reminder tabs in moments that seamlessly fit into users' activities. One approach is to estimate relevance between archived tabs and users' current activities and make decisions about when and how to resurface them. For example, a system may resurface a tab directly into a user's existing workflow (e.g. in a to-do list) or when a user is making similar search queries if the tab is highly relevant, or may resurface a tab within a user's existing activities in a more subtle way (e.g. in social media feed [33]). Effectively realizing this idea will require a system to monitor and predict current and future activities [36], and reason about the expected value of surfacing archived tabs at different moments by considering the relevance and potential disruption [26] to a users' existing workflows or activities.

Increasing the salience of tabs when they become more relevant. One of the main reasons participants kept their tabs open was to have quick access to frequently visited sources. While Google Chrome allows users to pin frequently visited tabs, pinned tabs actually become less salient with only favicons shown. This may work well with popular and well recognized single-page applications such as Gmail and Spotify, but less so when users have many references during online exploration tasks with less recognizable or identical favicons. In Study 1, we did not encounter extensive use of pinned tabs when we examined our interviewees' browser tabs.

Results from Study 1 also showed that the relevance of opened tabs might change over time as participants learned new information, which made it difficult for users to judge the future relevancy and importance of their tabs. For example, when a user is planning a trip to a new city, they may have many tabs open for hotels and restaurants. However, as they learn more about the city they may realize that hotels and restaurants in a certain neighborhood are too pricey for them and places in other neighborhoods become more relevant to them. Participants mentioned this difficulty in estimating the expected value of tabs and how they kept all potentially relevant tabs as a result of it. For example, one participant said they preferred to make decisions about which tabs were more important and relevant after having done their research and having built a better mental model of the information space:"I don't want to have to decide which things are important and throw things away while I am trying to research."

To ease the burden of users making explicit judgements on tab relevance, future systems need ways to better understand its users and their evolving interests [9], allowing it to increase saliency for tabs that became more relevant. Similar mechanisms have also been explored in the domain of graph data exploration and visualization [70] (i.e., by giving more salience to area with high degreeof-interest); perhaps these techniques could also be adapted to the browser to give more visual salience to tabs that were of interests to its users. For example, a system may estimate users' changing mental models based on their recent information consumption or external mental model representations (e.g., using lists or tables as mentioned in the previous section). This could enable the system to evaluate each tab's value to users (e.g., using information-theoretic estimators such as Good-Turing [51]); and adjust the salience of tabs accordingly, allowing browsers to intelligently assist users in drawing their attention to sources that contained information most valuable to them.

\section{LIMITATIONS}

Our sample of participants focused on 10 academic researchers supplemented by 103 crowdworkers with a wider range of occupations. While this allowed us to both gain deep qualitative insights from interviewing participants who are frequently engaged with online research and to generalize our findings with a larger scale survey, it is possible that a different population would lead to usage themes not discovered in this paper. 
Furthermore, our work primarily probed desktop contexts, and it is possible that mobile browser tabs may involve different usage patterns due to differences in screen real-estate and memory constraints as well as mobile contexts. Although some of our design implications may generalize to the mobile context (for example, task compartmentalization and resumption could also be important on mobile due to short bursts of use and frequent interruptions [32]), further studies are required to better understand issues users face when conducting tabbed browsing on mobile devices.

Finally, Studies 1 and 2 were conducted before the COVID-19 pandemic [11], while the exploratory interviews described in the Design Implication Section were conducted during the pandemic. While participants in exploratory interviews more frequently engaged in pandemic-related tasks (e.g., product research for supporting working from home or remote education), there were no noticeable differences in their tab browsing behavior patterns.

\section{CONCLUSIONS}

In this paper, we revisited today's tabbed browsing user behavior a decade after the previous in-depth study [15]. With the increase in scope and complexity of online tasks in the past decade, there has been growing evidence that a significant segment of users now face issues managing their tabs, commonly referred to as"tab overload". To better understand this, we focused our investigation on the challenges users face using browser tabs to conduct various tasks on the Web today. For this, we conducted a two-week-long interview study with ten researchers, a survey with 103 diverse participants, and exploratory design interviews with seven additional participants. Based on our findings, we proposed a user model with various opposing pressures for keeping tabs open and closing them, providing insights and explanations into why tab management can be challenging for users today. Based on our user model, we developed a set of design implications for future systems that can relieve these challenges.

Novel browser designs that can better support tab management are likely to become more important as users increasingly rely on browsing the Web to support a wide range of information, communication, productivity, and entertainment needs. Further, as social media, content providers, and advertisers are becoming more intelligent in using computational models to compete for users' attention, we need better technologies and designs to ensure users can focus on tasks that are important to them without being constantly distracted. We believe the user models and design implications developed in this work can inform the future designs of browser interfaces that would allow users to allocate their attention effectively, construct rich external mental models, and do so with little pressures from managing the many webpages opened in their browsers.

\section{ACKNOWLEDGMENTS}

This work was supported by the National Science Foundation (PFI1701005, FW-HTF-RL-1928631, and SHF-1814826), the Office of Naval Research, Google, and the Carnegie Mellon University Center for Knowledge Acceleration.

\section{REFERENCES}

[1] alan d baddeley and graham hitch. 1974. working memory. psychology of learning and motivation 8 (1974), 47-89.
[2] jeremy birnholtz, graham dixon, and jeffrey hancock. 2012. distance, ambiguity and appropriation: structures affording impression management in a collocated organization. computers in human behavior 28, 3 (2012), 1028-1035.

[3] Richard E Boyatzis. 1998. Transforming qualitative information: Thematic analysis and code development. sage publications, inc, Thousand Oaks, California, United States.

[4] Andrew Bragdon, Robert Zeleznik, Steven P Reiss, Suman Karumuri, William Cheung, Joshua Kaplan, Christopher Coleman, Ferdi Adeputra, and Joseph J LaViola Jr. 2010. Code bubbles: a working set-based interface for code understanding and maintenance. In Proceedings of the SIGCHI Conference on Human Factors in Computing Systems. ACM, New York, NY, USA, 2503-2512.

[5] Stuart K Card, George G Robertson, and William York. 1996. The WebBook and the Web Forager: an information workspace for the World-Wide Web. In Proceedings of the SIGCHI conference on Human factors in computing systems. ACM, New York, NY, USA, 111-ff.

[6] lara d catledge and james e pitkow. 1995. characterizing browsing strategies in the world-wide web. computer networks and isdn systems 27, 6 (1995), 1065-1073.

[7] Joseph Chee Chang, Nathan Hahn, and Aniket Kittur. 2016. Supporting Mobile Sensemaking Through Intentionally Uncertain Highlighting. In Proceedings of the 29th Annual Symposium on User Interface Software and Technology (Tokyo, Japan) (UIST '16). ACM, New York, NY, USA, 61-68. https://doi.org/10.1145/ 2984511.2984538

[8] Joseph Chee Chang, Nathan Hahn, and Aniket Kittur. 2020. Mesh: Scaffolding Comparison Tables for Online Decision Making. In Proceedings of the 33rd Annual ACM Symposium on User Interface Software and Technology (Virtual Event, USA) (UIST '20). Association for Computing Machinery, New York, NY, USA, 391-405. https://doi.org/10.1145/3379337.3415865

[9] Joseph Chee Chang, Nathan Hahn, Adam Perer, and Aniket Kittur. 2019. SearchLens: Composing and capturing complex user interests for exploratory search. In Proceedings of the 24th International Conference on Intelligent User Interfaces. ACM, New York, NY, USA, 498-509.

[10] Joseph Chee Chang, Aniket Kittur, and Nathan Hahn. 2016. Alloy: Clustering with Crowds and Computation. In Proceedings of the 2016 CHI Conference on Human Factors in Computing Systems (Santa Clara, California, USA) (CHI '16). ACM, New York, NY, USA, 3180-3191. https://doi.org/10.1145/2858036.2858411

[11] Gerardo Chowell and Kenji Mizumoto. 2020. The COVID-19 pandemic in the USA: what might we expect? The Lancet 395, 10230 (2020), 1093-1094.

[12] Shelia R Cotten and Sipi S Gupta. 2004. Characteristics of online and offline health information seekers and factors that discriminate between them. Social science \&amp; medicine 59, 9 (2004), 1795-1806.

[13] Robert DeLine and Kael Rowan. 2010. Code canvas: zooming towards better development environments. In Proceedings of the 32nd ACM/IEEE International Conference on Software Engineering-Volume 2. ACM, New York, NY, USA, 207-210.

[14] Jacob Devlin, Ming-Wei Chang, Kenton Lee, and Kristina Toutanova. 2019. BERT: Pre-training of Deep Bidirectional Transformers for Language Understanding. In Proceedings of the 2019 Conference of the North American Chapter of the Association for Computational Linguistics: Human Language Technologies, Volume 1 (Long and Short Papers). Association for Computational Linguistics, Stroudsburg, Pennsylvania, United States, 4171-4186.

[15] Patrick Dubroy and Ravin Balakrishnan. 2010. A Study of Tabbed Browsing Among Mozilla Firefox Users. In Proceedings of the SIGCHI Conference on Human Factors in Computing Systems (Atlanta, Georgia, USA) (CHI '10). ACM, New York, NY, USA, 673-682. https://doi.org/10.1145/1753326.1753426

[16] Chrome Extension. 2017. TabSuspender. https://chrome.google.com/webstore/ detail/ tab-suspender/ fiabciakcmgepblmdkmemdbbkilneeeh. Accessed: 2017-0910.

[17] Chrome Extension. 2017. TheGreatSuspender. https://chrome.google.com/ webstore/detail/the-great-suspender/klbibkeccnjlkjkiokjodocebajanakg. Accessed: 2017-09-10.

[18] Chrome Extension. 2017. vTabs. https://chrome.google.com/webstore/detail/vtabs/ okpnlgbgcfchbicbhjmmhldhkbkfilce. Accessed: 2017-09-10.

[19] Chrome Extension. 2020. OneTab. https://chrome.google.com/webstore/detail/ onetab/chphlpgkkbolifaimnlloiipkdnihall. Accessed: 2020-09-10.

[20] Chrome Extension. 2020. Workona. http://workona.com/. Accessed: 2020-09-15.

[21] Chrome Extension. 2021. Save to Pocket. https://chrome.google.com/webstore/ detail/save-to-pocket/niloccemoadcdkdjlinkgdfekeahmflj. Accessed: 2021-01-07.

[22] Chrome Extension. 2021. Search All Tabs. https://chrome.google.com/webstore/ detail/search-all-tabs/ndfnfhnlgoocpbbjjjfmiojdfcgcfomn . Accessed: 2021-01-08.

[23] Chrome Extension. 2021. SessionBuddy. https://chrome.google.com/webstore/ detail/session-buddy/edacconmaakjimmfgnblocblbcdcpbko. Accessed: 2021-0107.

[24] Chrome Extension. 2021. Toby. https://chrome.google.com/webstore/detail/ toby-for-chrome/hddnkoipeenegfoeaoibdmnaalmgkpip. Accessed: 2021-01-07.

[25] Firefox Extension. 2017. Firefox Extension: Tree Style Tabs. https://addons.mozilla.org/en-US/firefox/addon/tree-style-tab/?src=search. Accessed: 2017-09-10 
[26] James Fogarty, Scott E Hudson, Christopher G Atkeson, Daniel Avrahami, Jodi Forlizzi, Sara Kiesler, Johnny C Lee, and Jie Yang. 2005. Predicting human interruptibility with sensors. ACM Transactions on Computer-Human Interaction (TOCHI) 12, 1 (2005), 119-146.

[27] Susannah Fox, Lee Rainie, J Horrigan, A Lenhart, T Spooner, M Burke, O Lewis, and C Carter. 2000. The online health care revolution: How the Web helps Americans take better care of themselves. Pew Internet and American live project: Online life report. Retrieved July 21, 2005.

[28] Life Hacker. 2012. Master Your Browsers Tabs with These Tricks and Ex tensions. http://lifehacker.com/5883299/master-your-browsers-tabs-with-thesetricks-and-extensions. Accessed: 2017-09-10.

[29] Life Hacker. 2013. It's Okay to Open More Than Nine Browser Tabs; Here's How to Easily Manage Them. http://lifehacker.com/5985462/its-okay-to-open-more than-nine-browser-tabs-you-just-need-to-manage-them-properly. Accessed 2017-09-10.

[30] Life Hacker. 2013. Why You Should Never Have More Than Nine Browser Tabs Open. http://lifehacker.com/5984149/why-you-should-never-have-more-thannine-browser-tabs-open. Accessed: 2017-09-10.

[31] Nathan Hahn, Joseph Chang, Ji Eun Kim, and Aniket Kittur. 2016. The Knowledge Accelerator: Big Picture Thinking in Small Pieces. In Proceedings of the 2016 CHI Conference on Human Factors in Computing Systems (Santa Clara, California, USA) (CHI '16). ACM, New York, NY, USA, 2258-2270. https://doi.org/10.1145/2858036. 2858364

[32] nathan hahn, joseph chee chang, and aniket kittur. 2018. bento browser: complex mobile search without tabs. In proceedings of the 2018 chi conference on human factors in computing systems. ACM, New York, NY, USA, 1-12.

[33] Nathan Hahn, Shamsi T. Iqbal, and Jaime Teevan. 2019. Casual Microtasking. In Proceedings of the 2019 CHI Conference on Human Factors in Computing Systems - CHI '19. ACM Press, New York, NY, USA, 9. https://doi.org/10.1145/3290605. 3300249

[34] Austin Z Henley and Scott D Fleming. 2014. The Patchworks code editor: Toward faster navigation with less code arranging and fewer navigation mistakes. In Proceedings of the SIGCHI Conference on Human Factors in Computing Systems. ACM, New York, NY, USA, 2511-2520.

[35] E Tory Higgins. 1989. Self-discrepancy theory: What patterns of self-beliefs cause people to suffer? In Advances in experimental social psychology. Vol. 22. Elsevier, Amsterdam, Netherlands, 93-136.

[36] Eric Horvitz and Johnson Apacible. 2003. Learning and Reasoning about Interrup tion. In Proceedings of the 5th International Conference on Multimodal Interfaces (Vancouver, British Columbia, Canada) (ICMI '03). Association for Computing Machinery, New York, NY, USA, 20-27. https://doi.org/10.1145/958432.958440

[37] jeff huang, thomas lin, and ryen w white. 2012. no search result left behind branching behavior with browser tabs. In proceedings of the fifth acm international conference on web search and data mining. acm, acm, new york, ny, usa, 203-212.

[38] jeff huang and ryen w white. 2010. parallel browsing behavior on the web. In proceedings of the 21st acm conference on hypertext and hypermedia. acm, acm, new york, ny, usa, 13-18.

[39] Doist Inc. 2021. Todoist. https://todoist.com. Accessed: 2021-01-07.

[40] rosie jones and kristina lisa klinkner. 2008. beyond the session timeout: automatic hierarchical segmentation of search topics in query logs. In proceedings of the 17 th acm conference on information and knowledge management. acm, acm, new york, ny, usa, 699-708.

[41] aniket kittur, ed h chi, and bongwon suh. 2008. crowdsourcing user studies with mechanical turk. In proceedings of the sigchi conference on human factors in computing systems. acm, new york, ny, usa, 453-456.

[42] Aniket Kittur, Andrew M Peters, Abdigani Diriye, Trupti Telang, and Michael R Bove. 2013. Costs and benefits of structured information foraging. In Proceedings of the SIGCHI Conference on Human Factors in Computing Systems. ACM, ACM, New York, NY, USA, 2989-2998.

[43] Weize Kong, Michael Bendersky, Marc Najork, Brandon Vargo, and Mike Colagrosso. 2020. Learning to Cluster Documents into Workspaces Using Large Scale Activity Logs. In Proceedings of the 26th ACM SIGKDD International Conference on Knowledge Discovery and Data Mining (Virtual Event, CA, USA) (KDD '20). Association for Computing Machinery, New York, NY, USA, 2416-2424. https://doi.org/10.1145/3394486.3403291

[44] Minchu Kulkarni, Kshitij Kapoor, Deva Surya Vivek Madala, Sanchit Bansal, and Sudheendra Hangal. 2019. Compartmentalizing web browsing with Sailboat. In Proceedings of the 10th Indian Conference on Human-Computer Interaction. ACM, New York, NY, USA, 1-8.

[45] mark w lansdale. 1988. the psychology of personal information management applied ergonomics 19, 1 (1988), 55-66.

[46] Michael Xieyang Liu, Jane Hsieh, Nathan Hahn, Angelina Zhou, Emily Deng, Shaun Burley, Cynthia Taylor, Aniket Kittur, and Brad A Myers. 2019. Unakite: Scaffolding Developers' Decision-Making Using the Web. In Proceedings of the 32nd Annual ACM Symposium on User Interface Software and Technology. ACM New York, NY, USA, 67-80.

[47] Gary Marchionini. 2006. Exploratory search: from finding to understanding. Commun. ACM 49, 4 (2006), 41-46.
[48] Pablo N. Mendes, Max Jakob, Andrés García-Silva, and Christian Bizer. 2011. DBpedia Spotlight: Shedding Light on the Web of Documents. In Proceedings of the 7th International Conference on Semantic Systems (Graz, Austria) (I-Semantics '11). Association for Computing Machinery, New York, NY, USA, 1-8. https: //doi.org/10.1145/2063518.2063519

[49] dan morris, meredith ringel morris, and gina venolia. 2008. searchbar: a searchcentric web history for task resumption and information re-finding. In proceedings of the sigchi conference on human factors in computing systems. acm, acm, new york, ny, usa, 1207-1216.

[50] Hacker News. 2018. Open tabs are cognitive spaces (rybakov.com). https://news.ycombinator.com/?id=16671957. Accessed: 2020-09-13.

[51] Alon Orlitsky and Ananda Theertha Suresh. 2015. Competitive Distribution Estimation: Why is Good-Turing Good. In Advances in Neural Information Processing Systems, C. Cortes, N. Lawrence, D. Lee, M. Sugiyama, and R. Garnett (Eds.), Vol. 28. Curran Associates, Inc., 57 Morehouse Ln, Red Hook, NY 12571, United States, 2143-2151. https://proceedings.neurips.cc/paper/2015/file/ d759175de8ea5b1d9a2660e45554894f-Paper.pdf

[52] Jeffrey Pennington, Richard Socher, and Christopher D Manning. 2014. Glove: Global vectors for word representation. In Proceedings of the 2014 conference on empirical methods in natural language processing (EMNLP). Association for Computational Linguistics, Stroudsburg, Pennsylvania, United States, 1532-1543.

[53] David J Piorkowski, Scott D Fleming, Irwin Kwan, Margaret M Burnett, Christopher Scaffidi, Rachel KE Bellamy, and Joshua Jordahl. 2013. The whats and hows of programmers' foraging diets. In Proceedings of the SIGCHI Conference on Human Factors in Computing Systems. ACM, New York, NY, USA, 3063-3072.

[54] Peter Pirolli and Stuart Card. 1999. Information foraging. Psychological review 106, 4 (1999), 643

[55] The Open Directory Project. 1998-2017. DMOZ: The Open Directory Project. https://www.dmoz.org/. Accessed: 2017-09-10.

[56] Pradeep Racherla and Wesley Friske. 2012. Perceived 'usefulness' of online consumer reviews: An exploratory investigation across three services categories. Electronic Commerce Research and Applications 11, 6 (2012), 548-559.

[57] Reddit. 2013. I have a serious problem with browser tab hoarding. https://www.reddit.com/r/declutter/comments/1jpw13/ i_have_a_serious_problem_with_browser_tab_hoarding/. Accessed: 2017-09-10.

[58] Reddit. 2016. I'm a digital hoarder. I opened chrome to find all my tabs gone. I feel relieved. https://www.reddit.com/r/declutter/comments/4qkomc/ im_a_digital_hoarder_i_opened_chrome_to_find_all/. Accessed: 2017-09-10.

[59] George Robertson, Mary Czerwinski, Kevin Larson, Daniel C Robbins, David Thiel, and Maarten Van Dantzich. 1998. Data mountain: using spatial memory for document management. In Proceedings of the 11th annual ACM symposium on User interface software and technology. ACM, New York, NY, USA, 153-162.

[60] Barry Schwartz. 2004. The paradox of choice.

[61] Barry Schwartz, Andrew Ward, John Monterosso, Sonja Lyubomirsky, Katherine White, and Darrin R Lehman. 2002. Maximizing versus satisficing: happiness is a matter of choice. Fournal of personality and social psychology 83, 5 (2002), 1178.

[62] Anselm Strauss and Juliet Corbin. 1998. Basics of qualitative research: Techniques and procedures for developing grounded theory. sage publications, inc, Thousand Oaks, California, United States.

[63] Bin Tan and Fuchun Peng. 2008. unsupervised query segmentation using generative language models and wikipedia. In Proceedings of the 17th international conference on World Wide Web. ACM, New York, NY, USA, 347-356.

[64] linda tauscher and saul greenberg. 1997. how people revisit web pages: empirical findings and implications for the design of history systems. international journal of human-computer studies 47, 1 (1997), 97-137.

[65] Vivaldi Technologies. 2020. Vivaldi Browser. https://vivaldi.com/. Accessed: 2020-09-10.

[66] Jaime Teevan, Eytan Adar, Rosie Jones, and Michael AS Potts. 2007. Information re-retrieval: repeat queries in Yahoo's logs. In Proceedings of the 30th annual international ACM SIGIR conference on Research and development in information retrieval. ACM, New York, NY, USA, 151-158.

[67] Jaime Teevan, Christine Alvarado, Mark S Ackerman, and David R Karger. 2004. The perfect search engine is not enough: a study of orienteering behavior in directed search. In Proceedings of the SIGCHI conference on Human factors in computing systems. ACM, New York, NY, USA, 415-422.

[68] amos tversky and daniel kahneman. 1991. loss aversion in riskless choice: a reference-dependent model. the quarterly journal of economics 106, 4 (1991), 1039-1061.

[69] Chrome Product Updates. 2020. Keep tabs on your tabs in Google Chrome. https://blog.google/products/chrome/manage-tabs-with-google-chrome/. Accessed: 2020-09-15.

[70] Frank Van Ham and Adam Perer. 2009. "Search, show context, expand on demand": Supporting large graph exploration with degree-of-interest. IEEE Transactions on Visualization and Computer Graphics 15, 6 (2009), 953-960.

[71] Francesco Vitale, Izabelle Janzen, and Joanna McGrenere. 2018. Hoarding and Minimalism: Tendencies in Digital Data Preservation. In Proceedings of the 2018 CHI Conference on Human Factors in Computing Systems (Montreal QC, Canada) (CHI '18). Association for Computing Machinery, New York, NY, USA, 1-12. 
https://doi.org/10.1145/3173574.3174161

[72] W3C, W3Counter. 2017. Web Browser Usage Trends. https://www.w3 counter.com/trends. Accessed: 2017-09-10.

[73] harald weinreich, hartmut obendorf, eelco herder, and matthias mayer. 2006. off the beaten tracks: exploring three aspects of web navigation. In proceedings of the 15th international conference on world wide web. acm, ACM, New York, NY, USA, 133-142.

[74] harald weinreich, hartmut obendorf, eelco herder, and matthias mayer. 2008. not quite the average: an empirical study of web use. acm transactions on the web (tweb) 2, 1 (2008), 5.
[75] Mozilla Wiki. 2020. Firefox Containers. https://wiki.mozilla.org/Security/ Contextual_Identity_Project/Containers. Accessed: 2021-01-08.

[76] Alex C. Williams, Gloria Mark, Kristy Milland, Edward Lank, and Edith Law. 2019. The Perpetual Work Life of Crowdworkers: How Tooling Practices Increase Fragmentation in Crowdwork. Proc. ACM Hum.-Comput. Interact. 3, CSCW, Article 24 (Nov. 2019), 28 pages. https://doi.org/10.1145/3359126

[77] max 1 wilson et al. 2008. improving exploratory search interfaces: adding value or information overload?. In Second Workshop on Human-Computer Interaction and Information Retrieval. Microsoft Research, Redmond, WA, USA, 81-84. 